TRANSACTIONS OF THE

AMERICAN MATHEMATICAL SOCIETY

Volume 358, Number 8, Pages 3651-3671

S 0002-9947(06)04057-8

Article electronically published on March 24, 2006

\title{
GEOMETRIC STRUCTURES AS DEFORMED INFINITESIMAL SYMMETRIES
}

\author{
ANTHONY D. BLAOM
}

\begin{abstract}
A general model for geometric structures on differentiable manifolds is obtained by deforming infinitesimal symmetries. Specifically, this model consists of a Lie algebroid, equipped with an affine connection compatible with the Lie algebroid structure. The curvature of this connection vanishes precisely when the structure is locally symmetric.

This model generalizes Cartan geometries, a substantial class, to the intransitive case. Simple examples are surveyed and corresponding local obstructions to symmetry are identified. These examples include foliations, Riemannian structures, infinitesimal $G$-structures, symplectic and Poisson structures.
\end{abstract}

\section{INTRODUCTION}

According to Élie Cartan, a geometric structure is a symmetry deformed by curvature. Here we describe a model for geometric structures promoting this vision, formulated in the language of Lie algebroids.

If by symmetry we mean a smooth action $G_{0} \times M \rightarrow M$ of a Lie group $G_{0}$ on a smooth manifold $M$, then every symmetry has an infinitesimal counterpart: the corresponding action $\mathfrak{g}_{0} \times M \rightarrow T M$ of the Lie algebra $\mathfrak{g}_{0}$ of $G_{0}$. Such infinitesimal actions are generalized by vector bundle morphisms $\mathfrak{g} \rightarrow T M$, where $\mathfrak{g}$ is a possibly non-trivial vector bundle over $M$ known as a Lie algebroid. We call a Lie algebroid equipped with an affine connection $\nabla$ a Cartan algebroid whenever $\nabla$ is compatible with the Lie algebroid structure in an appropriate sense. As it turns out, one may then view the curvature of such a connection as the local obstruction to symmetry.

The significance of Cartan algebroids is that they are a natural model for many geometric structures. In a sense, they are infinitesimal versions of Cartan's espace generalisé, also known as Cartan geometries. Cartan geometries include first order structures, such as Riemannian and almost Hermitian structures, and higher order structures, such as projective, conformal and CR structures. For some details and further examples, see [16] and [17.

The present generalization adds intransitive structures (deformations of intransitive symmetries) to the list. These include foliations and Poisson manifolds, equipped with suitable connections. Even in the transitive case however, the Cartan algebroid point of view is somewhat novel.

Received by the editors April 28, 2004.

2000 Mathematics Subject Classification. Primary 53C15, 58H15; Secondary 53B15, 53C07, $53 \mathrm{C} 05,58 \mathrm{H} 05$.

Key words and phrases. Lie algebroid, geometric structure, Cartan geometry, Cartan connection, action Lie algebroid, deformation, connection theory.

(C)2006 American Mathematical Society
Reverts to public domain 28 years from publication 
For instance, 'curvature' assumes a new meaning. Classical notions of curvature measure deviation from a particular symmetric model. In a Cartan geometry, this model is a prescribed homogeneous space $G / H$. In the context of $G$-structures (see, e.g., [12]), the implicit model is usually $\mathbb{R}^{n}$.

By contrast, the present theory has no models: all symmetric structures are created equal and the curvature of $\nabla$ merely measures deviation from some symmetric structure. For example, Euclidean space, hyperbolic space and spheres are all regarded by us as 'flat' Riemannian structures.

Beyond the simplest of examples, however, the procedure by which a Cartan algebroid is associated with a given geometric structure is not trivial. This procedure amounts to a model-free version of Cartan's method of equivalence. For the $G$-structure implementation of this method, see, e.g., 9]. A Lie algebroid version of Cartan's method is developed in detail in our paper [2, which also refines or improves several ideas introduced in the present work.

Paper outline. In Sect.2 we recall how Lie algebroids may be viewed as generalized Lie algebra actions. We then formulate what it means for an affine connection on a Lie algebroid to be compatible with the Lie algebroid structure (Definition 2.3). Such connections, which we call Cartan connections, are related to the classical connections of the same name (see Sect.77). Theorem A (Sect.2) which characterizes the locally symmetric Cartan algebroids is then easily established. This furnishes one answer to the question: When is an arbitrary Lie algebroid a so-called action (or transformation) Lie algebroid?

The remainder of the paper focuses on examples demonstrating the versatility of the model; in each example the corresponding implications of Theorem A are identified. This neatly unifies several results for the first time, although the results themselves are mostly known. More substantial applications are pursued in [2].

In Sect. 3 we discuss absolute parallelisms and the question: When is a differential manifold a Lie group? More generally, we turn to the question: When are the leaves of a foliation the orbits of some Lie group action? After describing the simplest scenario explicitly, we sketch how the question might be answered more generally. Known conditions for the maximal local homogeneity of a Riemannian manifold are recovered and then generalized in Theorem B (Sect.6) to those infinitesimal $G$-structures supporting a Cartan connection of 'reductive' type. In Sect. 3.4 we discuss the existence of finite-dimensional symmetries in the Poisson category, again restricting to the simplest scenario. This gives an answer (admittedly simple-minded) to the question: When is a Poisson manifold the dual of a Lie algebra?

Sect.4reviews the theory of connections from the Lie algebroid viewpoint, and in Sect. 5 we recall how the first jet bundle associated with a Lie algebroid is another Lie algebroid; we use this to explain the real meaning of compatibility mentioned above. Sect.5 concludes with a brief discussion of invariant differential operators associated with a Cartan algebroid. This appears to be closely related to the socalled 'tractor' or 'local twistor' calculus used in conformal geometry [4].

In Sect.7 we explain how Cartan algebroids generalize Cartan geometries. We formulate Theorem C, characterizing those Cartan geometries whose corresponding Cartan algebroids are locally symmetric, a notion weaker than local flatness of the Cartan geometry itself (indicating local coincidence with the prescribed model). 
Choosing a Cartan connection on a transitive Lie algebroid $\mathfrak{g}$ amounts to choosing a certain 'representation' of $\mathfrak{g}$ on itself; see Sect.6.1. In Sect.6.2 we reduce the existence of such a representation to the existence of a representation of $\mathfrak{g}$ on $T M$. (For infinitesimal $G$-structures, for example, such a representation is god-given.) Existence in general is not addressed.

The present work concerns infinitesimal symmetries exclusively. Lie group symmetries or Lie pseudogroups of symmetries are not discussed. Efficient tools for globalizing our results would be the groupoid versions of Lie's Fundamental Theorems [5] and the integrability results of Dazord [7].

Kirill Mackenzie has pointed out that the Kumpera-Spencer theory of Lie equations [13] is a model of geometric structures based on deformations. A related theory is that of Griffiths [10]. The relationship between these theories and the present framework has not been explored.

Background. Cartan geometries are not so well known, and their history is somewhat murky. Fortunately, a lot may be learned about them from Sharpe's beautiful book [16], one inspiration for the present work. Other recent discussions of Cartan geometries are [1] and [17.

Lie algebroids and Lie groupoids are presently the subject of considerable attention. One highlight is the recent generalization of Lie's Third Fundamental Theorem, due to Crainic and Fernandes [5]. Our main sources of information have been [3] and [14].

\section{DEFORMING SYMMETRIES}

2.1. Infinitesimal symmetries. We will be deforming infinitesimal symmetries. For us these are infinitesimal actions on a smooth connected manifold $M$ by a Lie algebra $\mathfrak{g}_{0}$, i.e., a Lie algebra homomorphism

$$
\mathfrak{g}_{0} \rightarrow \Gamma(T M)
$$

into the space of smooth vector fields on $M$. Only smooth actions will be of interest, i.e., those for which the corresponding action map $\mathfrak{g}_{0} \times M \stackrel{\#}{\longrightarrow} T M$ is smooth.

View $\mathfrak{g}_{0} \times M$ as a vector bundle over $M$ and use the same symbol, \#, to annotate the associated map of sections,

$$
\Gamma\left(\mathfrak{g}_{0} \times M\right) \stackrel{\#}{\longrightarrow} \Gamma(T M) .
$$

This map is an extension of (1) when we regard $\mathfrak{g}_{0}$ as the subspace of $\Gamma\left(\mathfrak{g}_{0} \times M\right)$ consisting of constant sections.

A key observation is that the Lie bracket on $\mathfrak{g}_{0}$ extends in a natural way to a Lie bracket on $\Gamma\left(\mathfrak{g}_{0} \times M\right)$ in a way making the extension (2) of (1) a Lie algebra homomorphism as well.

To obtain the new bracket, one first extends the bracket $[\cdot, \cdot]_{\mathfrak{g}_{0}}$ on $\mathfrak{g}_{0}$ in a trivial way: viewing sections of $\mathfrak{g}_{0} \times M$ as $\mathfrak{g}_{0}$-valued functions on $M$, define $\tau(X, Y)(m):=$ $[X(m), Y(m)]_{\mathfrak{g}_{0}}$ for sections $X$ and $Y$ of $\mathfrak{g}_{0} \times M$. If $\nabla$ denotes the canonical flat affine connection on $\mathfrak{g}_{0} \times M$, then the sought after bracket is defined by

$$
[X, Y]_{\mathfrak{g}_{0} \times M}:=\nabla_{\# X} Y-\nabla_{\# Y} X+\tau(X, Y), \quad X, Y \in \Gamma\left(\mathfrak{g}_{0} \times M\right) .
$$

Note that $\tau$ is just the unique extension of $[\cdot, \cdot]_{\mathfrak{g}_{0}}$ that is linear with respect to smooth functions $f$ on $M$. By contrast, $[\cdot, \cdot]_{\mathfrak{g}_{0} \times M}$ satisfies a Leibnitz property 
mimicking that of the Jacobi-Lie bracket on $\Gamma(T M)$ :

$$
[X, f Y]_{\mathfrak{g}_{0} \times M}=f[X, Y]_{\mathfrak{g}_{0} \times M}+d f(\# X) Y .
$$

2.2. Lie algebroids. Lie algebroids generalize the infinitesimal symmetries described above. By definition, a Lie algebroid is any vector bundle $\mathfrak{g}$ over $M$ (generalizing $\mathfrak{g}_{0} \times M$ above), together with a vector bundle morphism $\#: \mathfrak{g} \rightarrow T M$, called the anchor, and an $\mathbb{R}$-linear Lie bracket $[\cdot, \cdot]_{\mathfrak{g}}$ on $\Gamma(\mathfrak{g})$ making $\#: \Gamma(\mathfrak{g}) \rightarrow \Gamma(T M)$ into a Lie algebra homomorphism. Additionally, the bracket should be Leibnitz in the sense that

$$
[X, f Y]_{\mathfrak{g}}=f[X, Y]_{\mathfrak{g}}+d f(\# X) Y .
$$

A morphism $\phi: \mathfrak{g} \rightarrow \mathfrak{h}$ of Lie algebroids is a morphism of the underlying vector bundles (covering the identity) whose lift $\phi: \Gamma(\mathfrak{g}) \rightarrow \Gamma(\mathfrak{h})$ to sections is a homomorphism of Lie algebras. Additionally, one requires $\# \circ \phi=\#$.

The bundle $\mathfrak{g}_{0} \times M$ described in Sect.2.1 is called an action Lie algebroid. In analogy with this case, an arbitrary Lie algebroid $\mathfrak{g}$ is transitive if its anchor $\#: \mathfrak{g} \rightarrow$ $T M$ is surjective; if $\mathfrak{g}$ is intransitive, the (possibly singular) distribution \#( $\mathfrak{g}) \subset T M$ is nevertheless integrable, giving rise to a foliation by the orbits of the algebroid. A Lie algebroid $\mathfrak{g}$ is regular if the orbits have constant dimension, or equivalently, if the anchor has a subbundle of $\mathfrak{g}$ as kernel.

The tangent bundle $T M$ is itself a Lie algebroid over $M$, with the identity on $T M$ as anchor; every Lie algebra is a Lie algebroid over a single point. A Lie algebroid with trivial anchor is a bundle of Lie algebras. In particular, this applies to the kernel of the anchor of a regular Lie algebroid. See Sect.3 and the work cited above for further examples.

2.3. Cartan algebroids. The connection $\nabla$ appearing in Sect.2.1 is an instance of the following general notion, central to all that follows.

Definition. A Cartan connection on a Lie algebroid $\mathfrak{g}$ over $M$, with anchor \#, will be any affine connection $\nabla$ on $\mathfrak{g}$ that is compatible with its bracket $[\cdot, \cdot]_{\mathfrak{g}}$ in the following sense:

$$
\nabla_{V}[X, Y]_{\mathfrak{g}}=\left[\nabla_{V} X, Y\right]_{\mathfrak{g}}+\left[X, \nabla_{V} Y\right]_{\mathfrak{g}}+\nabla_{\bar{\nabla}_{Y} V} X-\nabla_{\bar{\nabla}_{X} V} Y,
$$

for all $X, Y \in \Gamma(\mathfrak{g})$ and $V \in \Gamma(T M)$. Here

$$
\bar{\nabla}_{X} V:=\# \nabla_{V} X+[\# X, V]_{T M}, \quad X \in \Gamma(\mathfrak{g}), V \in \Gamma(T M),
$$

where $[\cdot, \cdot]_{T M}$ denotes the Jacobi-Lie bracket on vector fields on $M$. A Cartan algebroid is a Lie algebroid equipped with a Cartan connection. A morphism of Cartan algebroids is simply a connection-preserving morphism of the underlying Lie algebroids.

Regarding (1): An affine connection on $\mathfrak{g}$ amounts to a splitting $J^{1} \mathfrak{g} \stackrel{s}{\leftarrow} \mathfrak{g}$ of a canonical exact sequence $0 \rightarrow T^{*} M \otimes \mathfrak{g} \hookrightarrow J^{1} \mathfrak{g} \rightarrow \mathfrak{g} \rightarrow 0$. Here $J^{1} \mathfrak{g}$ denotes the first jet bundle of $\mathfrak{g}$. As we elaborate in Sect. 5 each arrow is a morphism of Lie algebroids and condition (1) holds precisely when $J^{1} \mathfrak{g} \stackrel{s}{\leftarrow} \mathfrak{g}$ is a Lie algebroid morphism also.

The operator $\bar{\nabla}$ is an example of $\mathfrak{g}$-connection on $T M$; see Sect.4.4. The $\mathfrak{g}$ connection is flat if

$$
\left(\bar{\nabla}_{X} \bar{\nabla}_{Y}-\bar{\nabla}_{Y} \bar{\nabla}_{X}-\bar{\nabla}_{[X, Y]_{\mathfrak{g}}}\right) V=0
$$


for all $X, Y \in \Gamma(\mathfrak{g})$ and $V \in \Gamma(T M)$. Flat $\mathfrak{g}$-connections are called $\mathfrak{g}$-representations because they generalize the usual representations of a Lie algebra; instead of acting on a vector space, Lie algebroids act on vector bundles, in this case $T M$.

If $\nabla$ is to be a Cartan connection, then it is necessary that $\bar{\nabla}$ be a $\mathfrak{g}$-representation. One sees this by applying the anchor \# to both sides of (11). If $\#: \mathfrak{g} \rightarrow T M$ is injective, this condition is sufficient. (For examples, see Sects.3.1 and 3.2.) Lie algebroid representations are reviewed in Sect.4.

From a Cartan connection $\nabla$ one also obtains a representation of $\mathfrak{g}$ on itself, with respect to which the anchor \# is equivariant. See Sect.5.3. This is significant because, unlike Lie algebras, a Lie algebroid does not generally admit an adjoint representation in the naive sense (but do see Sect. [5.2).

2.4. The symmetric part of a Cartan algebroid. A fundamental observation is that every Cartan algebroid $(\mathfrak{g}, \nabla)$ has a canonical subalgebroid isomorphic to an action Lie algebroid. Indeed, let $\mathfrak{g}_{0} \subset \Gamma(\mathfrak{g})$ be the subspace of $\nabla$-parallel sections, which is finite dimensional. Then bracket compatibility 2.3(1) guarantees that $\mathfrak{g}_{0} \subset \Gamma(\mathfrak{g})$ is a Lie subalgebra, and we obtain an action of $\mathfrak{g}_{0}$ on $M$ given by

$$
\begin{gathered}
\mathfrak{g}_{0} \times M \rightarrow T M, \\
(X, m) \mapsto \# X(m) .
\end{gathered}
$$

Equipping $\mathfrak{g}_{0} \times M$ with the canonical flat affine connection, we obtain a morphism of Cartan algebroids,

$$
\begin{gathered}
\mathfrak{g}_{0} \times M \rightarrow \mathfrak{g}, \\
(X, m) \mapsto X(m) .
\end{gathered}
$$

This morphism is injective because $\nabla$-parallel sections vanishing at a point vanish everywhere. (We are assuming $M$ is connected.) We call the image of the monomorphism (11) the symmetric part of $(\mathfrak{g}, \nabla)$.

2.5. Curvature as the local obstruction to symmetry. A Cartan algebroid $(\mathfrak{g}, \nabla)$ on $M$ is symmetric if it is isomorphic to an action algebroid $\mathfrak{g}_{0} \times M$, equipped with its canonical flat connection - or equivalently, if it coincides with its symmetric part. We call $(\mathfrak{g}, \nabla)$ locally symmetric if every point of $M$ has an open neighborhood $U$ on which the restriction $\left.(\mathfrak{g}, \nabla)\right|_{U}$ is symmetric.

Cartan algebroids are indeed symmetries deformed by curvature:

Theorem A. A Cartan algebroid $(\mathfrak{g}, \nabla)$ on $M$ is locally symmetric if and only if $\nabla$ is flat. When $M$ is simply connected, local symmetry already implies symmetry.

Proof. The necessity of flatness is immediate. To finish the proof it suffices to show that (11) is an isomorphism whenever $\nabla$ is flat and $M$ is simply connected. Indeed, in that case $\nabla$ determines a trivialization of the bundle $\mathfrak{g}$ in which constant sections correspond to the $\nabla$-parallel sections of $\mathfrak{g}$-that is, to elements of $\mathfrak{g}_{0}$. (This classical result follows, for example, from the groupoid version of Lie's Second Fundamental Theorem; see Remark 4.5(3).) In particular, $\mathfrak{g}_{0} \times M$ and $\mathfrak{g}$ will have the same rank, implying the monomorphism (11) is an isomorphism.

\section{ExAmples}

We now describe simple examples of Cartan algebroids and describe simple consequences of Theorem A, stated as corollaries. More general classes of examples are presented in Sects. [6] and 7. 
3.1. Absolute parallelisms. The simplest application of Theorem A is to the question: When is a manifold a Lie group?

Affine connections on a tangent bundle $T M$ occur in pairs. If $\nabla$ is one such connection, its dual $\nabla^{*}$ is defined by

$$
\nabla_{X}^{*} Y:=\nabla_{Y} X+[X, Y]_{T M} .
$$

We have $\nabla^{* *}=\nabla$ and call $\left(\nabla, \nabla^{*}\right)$ a dual pair. When $M$ is a Lie group, the flat connections associated with the canonical left and right trivializations of the tangent bundle $T M$ are a dual pair in this sense. Conversely, any dual pair of simultaneously flat connections determines a local Lie group structure, as we now explain.

A Cartan connection on $T M$ is simply an affine connection whose dual is flat. This follows from comments made in Sect.2.3 (where $\nabla^{*}=\bar{\nabla}$ ). So specifying a Cartan connection is equivalent to specifying a flat affine connection on $T M$. These latter connections are the infinitesimal analogues of absolute parallelisms (trivializations of the tangent bundle), the most basic of all geometric structures.

Specifying an absolute parallelism on $M$ is equivalent to specifying a one-form $\omega$ on $M$, taking values in some vector space $V$, such that $\omega: T_{m} M \rightarrow V$ is an isomorphism at all $m \in M$.

Corollary. Let $\left(\nabla, \nabla^{*}\right)$ be a dual pair of connections on the tangent bundle of a simply connected manifold $M$ and suppose $\nabla$ and $\nabla^{*}$ are both flat. Then there exists a Lie algebra $\mathfrak{g}_{0}$ and an absolute parallelism $\omega \in \Omega^{1}\left(M, \mathfrak{g}_{0}\right)$ such that

$$
\begin{gathered}
d \omega+\frac{1}{2}[\omega, \omega]_{\mathfrak{g}_{0}}=0, \\
\omega\left(\nabla_{X} Y\right)=\left(\mathcal{L}_{X} \omega\right)(Y) \quad \text { and } \omega\left(\nabla_{X}^{*} Y\right)=\mathcal{L}_{X}(\omega(Y)) .
\end{gathered}
$$

Here $\mathcal{L}$ denotes Lie derivative.

If in addition the 'Mauer-Cartan' form $\omega$ is complete, then $M$ is diffeomorphic to the simply connected Lie group $G_{0}$ integrating $\mathfrak{g}_{0}$; see [16, Theorem 3.8.7] or [9].

Remark. If $\nabla^{*}$ is flat, then $\nabla$ is also flat if and only if the torsion of $\nabla^{*}$ is $\nabla^{*}$-parallel (Proposition 4.6(3) ).

The corollary is established by taking $\mathfrak{g}=T M$ in Theorem A, which then delivers an isomorphism $T M \rightarrow \mathfrak{g}_{0} \times M$. One takes $\omega$ to be the composite $T M \rightarrow \mathfrak{g}_{0} \times M \rightarrow$ $\mathfrak{g}_{0}$.

3.2. Foliations. It is natural to ask when the leaves of an arbitrary foliation $\mathcal{F}$ are the orbits of some action by a Lie group, or at least a Lie algebra. We now mention the most elementary application of Theorem A to this question.

Let $D \subset T M$ be a subbundle that is integrable as a distribution. Then $D \subset T M$ is a Lie subalgebroid. An affine connection $\nabla$ on $D$ is a Cartan connection precisely when the corresponding $D$-connection $\bar{\nabla}$, defined by

$$
\bar{\nabla}_{X} V:=\nabla_{V} X+[X, V]_{T M}, \quad X \in \Gamma(D), V \in \Gamma(T M),
$$

is flat. Call a Lie algebra action free if all orbits have the dimension of the Lie algebra. Theorem A implies:

Corollary. Let $\mathcal{F}$ be a regular foliation on a simply connected manifold $M$, and let $D \subset T M$ denote its tangent distribution. Then the orbits of $\mathcal{F}$ are the orbits of 
a free Lie algebra action if and only if $D$ admits a flat affine connection $\nabla$ whose corresponding D-connection $\bar{\nabla}$ is also flat.

Taking $D=T M$, we recover the preceding corollary, since the existence of a Mauer-Cartan form is nothing more than the existence of a free and transitive action by a Lie algebra.

More generally, one may look for Lie algebra actions of 'higher order' and consider smooth foliations $\mathcal{F}$ with singularities. Call an action by a Lie algebra $\mathfrak{g}_{0} k$ th-order faithful if the natural morphism $\mathfrak{g}_{0} \times M \rightarrow J^{k}(T M)$ into the $k$ th-order jet bundle of $T M$ is injective, i.e., realizes $\mathfrak{g}_{0} \times M$ as a subalgebroid of $J^{k}(T M)$. If $D \subset T M$ is the distribution tangent to a possibly singular foliation $\mathcal{F}$, then one searches for subalgebroids $\mathfrak{g} \subset J^{k}(T M)$ with $\#(\mathfrak{g})=D$, and establishes ' $k$ th-order symmetry' of $\mathcal{F}$ by finding a flat Cartan connection on $\mathfrak{g}$. In the case $D=T M$, obstructions in this search represent obstructions to the realization of $M$ as a homogenous space.

3.3. Riemannian structures. Let $\sigma$ be a Riemannian metric on $M$. Then an infinitesimal isometry of $(M, \sigma)$, or Killing field, is a vector field $V$ on $M$ such that $\mathcal{L}_{V} \sigma=0$, where $\mathcal{L}$ is Lie derivative. That is,

$$
\left(\mathcal{L}_{V} \sigma\right)(U, W):=\mathcal{L}_{V}(\sigma(U, W))-\sigma\left(\mathcal{L}_{V} U, W\right)+\sigma\left(U, \mathcal{L}_{V} W\right)
$$

Generally speaking, no Killing fields exist, even locally. However, for each fixed $m \in M$, there are many vector fields $V$ such that $\mathcal{L}_{V} \sigma$ vanishes at the point $m$. The one-jets $[V]_{m}^{1}$ at $m$ of all such $V$ are elements of a fundamental Lie algebroid $\mathfrak{g}$ associated with $\sigma$. In detail, if $J^{1}(T M)$ denotes the first jet bundle of $T M$ (a Lie algebroid, see Sect.5.1), then we define $\mathfrak{g}$ to be the kernel of

$$
\begin{aligned}
& J^{1}(T M) \rightarrow \operatorname{Sym}^{2}(T M), \\
& {[V]_{m}^{1} \mapsto\left(\mathcal{L}_{V} \sigma\right)(m) . }
\end{aligned}
$$

This is a transitive Lie subalgebroid of $J^{1}(T M)$. The anchor \# of $\mathfrak{g}$ is the restriction of the natural projection $J^{1}(T M) \rightarrow T M$. The kernel $\mathfrak{h} \subset T^{*} M \otimes T M \subset J^{1}(T M)$ of this anchor is the Lie algebra bundle of all $\sigma$-skew symmetric tangent space endomorphisms.

Because $\mathfrak{g} \subset J^{1}(T M)$ is transitive, there exists a (non-unique) splitting $\mathfrak{g} \leftarrow T M$ of the exact sequence $0 \rightarrow \mathfrak{h} \hookrightarrow \mathfrak{g} \stackrel{\#}{\rightarrow} T M \rightarrow 0$ such that $\mathfrak{g}=\mathfrak{h}+t(T M)$. For every such $t$, the composite $J^{1}(T M) \hookleftarrow \mathfrak{g} \leftarrow T M$ is a splitting of the exact sequence $0 \rightarrow T^{*} M \otimes T M \hookrightarrow J^{1}(T M) \rightarrow T M \rightarrow 0$, and so determines an affine connection $\nabla^{\mathrm{L}-\mathrm{C}}$ on $T M$. This is precisely the Levi-Cevita connection corresponding to $\sigma$ when $t$ is the unique splitting making $\nabla^{\mathrm{L}-\mathrm{C}}$ torsion free.

As we explain in Sect.5.2. there is a natural representation of $J^{1}(T M)$ on $T M$, denoted ad, and defined by $\operatorname{ad}_{J^{1} V} W=[V, W]_{T M}$, where $J^{1} V$ denotes the first prolongation of $V$. This representation restricts to a representation of $\mathfrak{g}$ on $T M$. As we detail in Sect.6.2, once a representation of $\mathfrak{g}$ on $T M$ is prescribed, a splitting $t$ as above determines an associated 'reductive' Cartan connection $\nabla$ on $\mathfrak{g}$. Choosing the splitting $t$ corresponding to the Levi-Cevita connection, we obtain a connection $\nabla$ with the following property.

Proposition. A vector field $V$ is a Killing field for $\sigma$ if and only if $V=\# X$ for some $\nabla$-parallel section $X$ of $\mathfrak{g}$ (equivalently, if $J^{1} V$ is a section of $\mathfrak{g}$ ). 
Sufficiency of the stated condition is not difficult to see. Its necessity may be established using Cartan's method of equivalence. See [2], which contains an alternative construction of $\nabla$ (there denoted $\left.\nabla^{(1)}\right)$.

The proposition establishes a correspondence between the Killing fields of $\sigma$ and the $\nabla$-parallel sections of $\mathfrak{g}$. It implies that $(M, \sigma)$ is locally maximally homogeneous, in the sense of locally possessing a Lie algebra of Killing fields of maximal dimension, if and only if $(\mathfrak{g}, \nabla)$ is locally symmetric, in the sense of Sect.2.5.

Corollary. The above Cartan algebroid $(\mathfrak{g}, \nabla)$ on a Riemannian manifold $(M, \sigma)$ is locally maximally homogeneous, if and only if the curvature of the Levi-Cevita connection is simultaneously $\mathfrak{h}$-invariant, and $\nabla^{\mathrm{L}-\mathrm{C}}$-parallel.

As is well known, the stated condition is equivalent to pure, constant scalar curvature, or equivalently, to constant sectional curvature. This corollary of Theorem A is a special case of Theorem B, p. 3668.

3.4. Symplectic and Poisson structures. Let $\omega$ be a symplectic structure on $M$ and let \#: $T^{*} M \rightarrow T M$ denote the inverse of $v \mapsto \omega(v, \cdot)$. Since \# is an isomorphism, there is a unique bracket on $\Gamma\left(T^{*} M\right)$ making $T^{*} M$ into a Lie algebroid with anchor \#. This bracket is given by

$$
[\alpha, \beta]_{T^{*} M}=\mathcal{L}_{\# \alpha} \beta-\mathcal{L}_{\# \beta} \alpha+d(\Pi(\alpha, \beta)), \quad \alpha, \beta \in \Gamma\left(T^{*} M\right),
$$

where $\mathcal{L}$ denotes Lie derivative and $\Pi$ is the Poisson tensor. This tensor is defined by $\Pi(\alpha, \beta):=\omega(\# \alpha, \# \beta)$ and so satisfies

$$
\langle\alpha, \# \beta\rangle=\Pi(\alpha, \beta) \quad \alpha, \beta \in \Gamma\left(T^{*} M\right) .
$$

As is well known, (11) defines a Lie algebroid structure on $T^{*} M$ for any Poisson manifold $(M, \Pi)$, with anchor \# defined by (2).

An infinitesimal isometry of a Poisson manifold $(M, \Pi)$ is a vector field $V$ on $M$ such that $\mathcal{L}_{V} \Pi=0$. Like foliations, Poisson manifolds have an abundance of infinitesimal isometries. In particular, every closed one-form $\alpha$ on $M$ determines an infinitesimal symmetry $\# \alpha$ tangent to the symplectic leaves known as a local Hamiltonian vector field, or a Hamiltonian vector field if $\alpha$ is exact.

The simplest instance of finite-dimensional symmetry occurs when $T^{*} M$ is itself isomorphic to an action Lie algebroid. This occurs, for instance, when $M$ is the dual of a Lie algebra, equipped with its Lie-Poisson structure [15, §10.1]. According to the corollary below, this is almost the only such case.

Let $\nabla$ be an affine connection on the Lie algebroid $T^{*} M$ associated with an arbitrary Poisson manifold $(M, \Pi)$. Assume that the space $\mathfrak{g}_{0}$ of $\nabla$-parallel sections consists of closed one-forms. It suffices to suppose $\nabla$ is torsion free, in the sense that the corresponding connection on $T M$ is torsion free; in the flat case this sufficient condition becomes necessary as well. Equivalently,

$$
d \alpha(V, W)=\left\langle\nabla_{V} \alpha, W\right\rangle-\left\langle\nabla_{W} \alpha, V\right\rangle,
$$

for all one-forms $\alpha$ and vector fields $V, W$ on $M$.

Assuming $\nabla$ is torsion free, we can readily use (3) to rewrite (11) as

$$
[\alpha, \beta]_{T^{*} M}=\nabla_{\# \alpha} \beta-\nabla_{\# \beta} \alpha-\nabla \Pi(\alpha, \beta),
$$

where $\nabla \Pi$ is the section of $\operatorname{Alt}^{2}\left(T^{*} M, T^{*} M\right)$ defined by

$$
\nabla \Pi(\alpha, \beta) V=\left(\nabla_{V} \Pi\right)(\alpha, \beta), \quad \alpha, \beta \in \Gamma\left(T^{*} M\right), V \in \Gamma(T M) .
$$


Using (4), it is not too difficult to characterize those torsion-free connections satisfying 2.3(1):

Lemma. A torsion-free connection $\nabla$ on $T^{*} M$ is a Cartan connection if and only if

$$
\operatorname{curv} \nabla(V, \# \alpha) \beta-\operatorname{curv} \nabla(V, \# \beta) \alpha-\left(\nabla_{V} \nabla \Pi\right)(\alpha, \beta)=0,
$$

for all one-forms $\alpha, \beta$ and vector fields $V$ on $M$.

We may now prove the following corollary of Theorem A, answering the question: When is a Poisson manifold the dual of a Lie algebra? Note that hypotheses (6) and (7) of the corollary are necessary in this case.

Corollary. Let $(M, \Pi)$ be a simply connected Poisson manifold. Assume:

(6) The tangent bundle TM admits a flat, torsion-free connection $\nabla$ such that $\nabla \Pi$ is $\nabla$-parallel.

Then the space $\mathfrak{g}_{0}$ of $\nabla$-parallel one-forms on $M$ is a finite-dimensional subalgebra of $\Gamma\left(T^{*} M\right)$, acting leaf-transitively on $M$ by Hamiltonian vector fields. Moreover assume:

(7) Some corresponding momentum map $\mathbf{J}: M \rightarrow \mathfrak{g}_{0}^{*}$ is proper and infinitesimally equivariant (see, e.g., [15]).

Then $\mathbf{J}$ maps $M$ isomorphically onto an open subset of $\mathfrak{g}_{0}^{*}$, equipped with its LiePoisson structure.

Proof. Assuming (6) holds, denote the corresponding affine connection on $T^{*} M$ by

$\nabla$ also. By the lemma, $\nabla$ is a Cartan connection on $T^{*} M$. That $\mathfrak{g}_{0} \subset \Gamma\left(T^{*} M\right)$ is a subalgebra acting on $M$ follows from observations made in Sect.2.4. Since $\nabla$ is torsion free and $M$ is simply connected, elements of $\mathfrak{g}_{0}$ are exact one-forms, so that $\mathfrak{g}_{0}$ acts via Hamiltonian vector fields. The action consequently admits a momentum map.

Since $\nabla$ is flat, the canonical inclusion $\mathfrak{g}_{0} \times M \hookrightarrow T^{*} M$ is an isomorphism (see the proof of Theorem A), implying that $\mathfrak{g}_{0}$ acts leaf-transitively and that any momentum map $\mathbf{J}: M \rightarrow \mathfrak{g}_{0}^{*}$ is a local diffeomorphism. Properness of $\mathbf{J}$ and simple connectivity of $M$ then imply $\mathbf{J}$ is a diffeomorphism onto its image. That $\mathbf{J}$ is additionally Poisson follows from its infinitesimal equivariance [15, §12.4].

\section{Connections and Lie algebroid Representations}

The next two sections expand on remarks made in Sect.2.3 and prepare us for the general classes of examples to be described in Sects.66and7 The present section reviews basic connection theory from the Lie algebroid point of view.

Lie algebroid formalism suggests a generalization of affine connections called $\mathfrak{g}$-connections, studied systematically in [8. (where they are the $A$-derivatives associated with $A$-connections). Just as Lie algebras act on vector spaces, so Lie algebroids act on vector bundles. A $\mathfrak{g}$-connection is just a Lie algebroid representation 'deformed by curvature' as we presently explain.

What we will describe as the 'general linear Lie algebroid $\mathfrak{g l}(E)$ ' of a vector bundle $E$ has been previously described in [11], where it is denoted $\operatorname{CDO}(E)$. 
4.1. Curvature. All the 'curvatures' in this paper are instances of a single Liealgebraic notion. Recall that a vector bundle morphism $\phi: \mathfrak{g} \rightarrow \mathfrak{h}$ between Lie algebroids is a Lie algebroid morphism if:

(1) $\# \circ \phi=\#$, and

(2) $\phi[X, Y]_{\mathfrak{g}}=[\phi X, \phi Y]_{\mathfrak{h}}$ for all $X, Y \in \Gamma(\mathfrak{g})$.

When only (11) holds, we define the curvature of $\phi$ by

$$
\operatorname{curv} \phi(X, Y):=[\phi X, \phi Y]_{\mathfrak{h}}-\phi[X, Y]_{\mathfrak{g}} \quad X, Y \in \Gamma(\mathfrak{g}) .
$$

Thus $\phi$ is a Lie algebroid morphism if and only if $\operatorname{curv} \phi=0$. Note that curv $\phi(X, Y)$ always lies in the kernel of $\#: \mathfrak{h} \rightarrow T M$.

4.2. The general linear Lie algebroid of a vector bundle. A representation of a Lie algebra $\mathfrak{g}$ is a vector space $E$, together with a Lie algebra homomorphism

$$
\mathfrak{g} \rightarrow \mathfrak{g l}(E) .
$$

Here $\mathfrak{g l}(E):=\operatorname{Hom}(E, E)$ is the Lie algebra of the general linear group $\operatorname{GL}(E)=$ $\operatorname{Aut}(E)$. If instead $E$ is a vector bundle over $M$, then we use $\operatorname{GL}(E)$ to denote the frame groupoid of $E$, consisting of all isomorphisms $E_{m_{1}} \rightarrow E_{m_{2}}$ between fibers of $E$ over possibly distinct points $m_{1}, m_{2} \in M$. We define $\operatorname{Aut}(E) \subset \mathrm{GL}(E)$ to be the subgroupoid consisting of isomorphisms $E_{m_{1}} \rightarrow E_{m_{2}}$ with $m_{1}=m_{2}$ (the isotropy subgroupoid). This subgroupoid is a bundle of Lie groups.

We now describe, in purely algebraic terms, a concrete model for the Lie algebroid of $\mathrm{GL}(E)$, a model we will denote by $\mathfrak{g l}(E)$. Sections of $\mathfrak{g l}(E)$ will be characterized as differential operators. The Lie algebroid bracket of two sections will simply be the commutator of the corresponding operators.

Let $J^{1} E$ denote the first jet bundle of $E$ and let $J^{1}: \Gamma(E) \rightarrow \Gamma\left(J^{1} E\right)$ denote prolongation: $J^{1}(\sigma)(m):=[\sigma]_{m}^{1}$. Here $[\sigma]_{m}^{1}$ denotes the one-jet of $\sigma$ at $m$. We have an exact sequence

$$
0 \rightarrow T^{*} M \otimes E \hookrightarrow J^{1} E \rightarrow E \rightarrow 0,
$$

where the inclusion on the left is the one sending a section $d f \otimes \sigma$ of $T^{*} M \otimes E$ to the section $J^{1}(f \sigma)-f J^{1} \sigma$ of $J^{1} E$. Applying $\operatorname{Hom}(\cdot, E)$ to the sequence, and identifying $\operatorname{Hom}\left(T^{*} M \otimes E, E\right)$ with $T M \otimes \operatorname{Hom}(E, E)$, we obtain a second exact sequence

$$
0 \rightarrow \operatorname{Hom}(E, E) \hookrightarrow \operatorname{Hom}\left(J^{1} E, E\right) \stackrel{*}{\rightarrow} T M \otimes \operatorname{Hom}(E, E) \rightarrow 0 .
$$

Definition. The general linear Lie algebroid $\mathfrak{g l}(E)$ of a vector bundle $E$ over $M$ is the preimage, under the surjective arrow $*$, of the subbundle $T M \otimes\left\{\operatorname{id}_{E}\right\} \subset$ $T M \otimes \operatorname{Hom}(E, E)$.

The general linear Lie algebroid $\mathfrak{g l}(E)$ of $E$ is indeed a Lie algebroid, as we now explain. First, its anchor is the map \# completing the following commutative diagram:

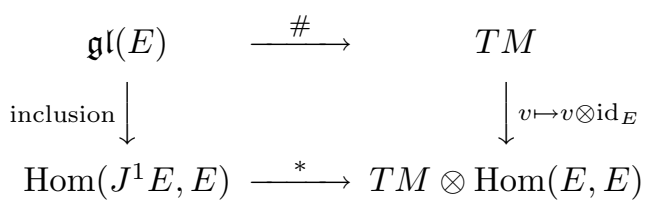


The bundle of Lie algebras $\operatorname{Hom}(E, E) \subset \operatorname{Hom}\left(J^{1} E, E\right)$ (the Lie algebroid of $\operatorname{Aut}(E))$ is contained in $\mathfrak{g l}(E)$. Moreover $\operatorname{Hom}(E, E)$ is precisely the kernel of the anchor \# above, i.e., we have an exact sequence

$$
0 \rightarrow \operatorname{Hom}(E, E) \hookrightarrow \mathfrak{g l}(E) \rightarrow \# T M \rightarrow 0 .
$$

For any section $\xi$ of $\operatorname{Hom}\left(J^{1} E, E\right)$, we define a first order differential operator, $\operatorname{oper}(\xi): \Gamma(E) \rightarrow \Gamma(E)$, by

$$
\operatorname{oper}(\xi) \sigma:=\xi\left(J^{1} \sigma\right)
$$

Unravelling the definitions above, one has:

Proposition. A section $\xi$ of $\operatorname{Hom}\left(J^{1} E, E\right)$ is a section of $\mathfrak{g l}(E)$ if and only if there exists a vector field $V$ on $M$ such that

$$
\operatorname{oper}(\xi)(f \sigma)=f \operatorname{oper}(\xi) \sigma+d f(V) \sigma
$$

for all smooth functions $f$ on $M$, and sections $\sigma$ of $E$. In that case, $V=\# \xi$.

A Lie algebroid bracket for $\mathfrak{g l}(E)$ is now well defined by

$$
\operatorname{oper}\left([\xi, \eta]_{\mathfrak{g r}(E)}\right)=\operatorname{oper}(\xi) \circ \operatorname{oper}(\eta)-\operatorname{oper}(\eta) \circ \operatorname{oper}(\xi) \text {. }
$$

By construction, the vector bundle $\operatorname{Hom}(E, E)$ is contained in $\mathfrak{g l}(E)$ as a Lie subalgebroid. The bracket $[\cdot, \cdot]_{\operatorname{Hom}(E, E)}$ that $\operatorname{Hom}(E, E)$ inherits from that on $\mathfrak{g l}(E)$ agrees with its usual Lie algebra bundle bracket:

$$
\left[\phi_{1}, \phi_{2}\right]_{\operatorname{Hom}(E, E)}=\phi_{1} \circ \phi_{2}-\phi_{2} \circ \phi_{1} \text {. }
$$

It can be shown that the Lie algebroid $\mathfrak{g l}(E)$ is indeed a model for the (abstract) Lie algebroid of $\mathrm{GL}(E)$. An element of the latter may be regarded as some tangent vector $\dot{g}(0) \in T \mathrm{GL}(E)$, where the path $t \mapsto g(t) \in \mathrm{GL}(E)$ consists of isomorphisms $g(t): E_{m(t)} \rightarrow E_{m}$ with $m(0)=m$ (i.e., is an 'infinitesimal moving $E$-frame'). The corresponding element of $\mathfrak{g l}(E) \subset \operatorname{Hom}\left(J^{1} E, E\right)$ sends $[\sigma]_{m}^{1} \in J^{1} E$ to $\left.\frac{d}{d t} g(t) \sigma(m(t))\right|_{t=0} \in E$.

4.3. $\mathfrak{g}$-representations. A representation of a Lie algebroid $\mathfrak{g}$ is a vector bundle $E$, together with a morphism of Lie algebroids, $\mathfrak{g} \rightarrow \mathfrak{g l}(E)$. If $\mathfrak{g}$ and $E$ are vector bundles over a single point, we obtain the usual notion of a Lie algebra representation. For another example, consider a principal bundle $P \rightarrow M$ with a simply connected structure group $H$, and corresponding Lie algebroid $\mathfrak{g}:=(T P) / H$, described in Sect.7.2. Then the representations of $\mathfrak{g}$ are the associated vector bundles of $P$; see, e.g., 14. In particular, there is in this case a one-to-one correspondence between (isomorphism classes of) representations of $\mathfrak{g}$ and vector space representations of $H$.

4.4. $\mathfrak{g}$-connections. Given a Lie algebroid $\mathfrak{g}$, a $\mathfrak{g}$-connection on a vector bundle $E$ is a morphism of vector bundles

$$
\nabla: \mathfrak{g} \rightarrow \mathfrak{g l}(E)
$$

satisfying $\# \circ \nabla=\#$ that is possibly not a Lie algebroid morphism. Recall that $\mathfrak{g l}(E) \subset \operatorname{Hom}\left(J^{1} E, E\right)$. For any vector bundle morphism

$$
\nabla: \mathfrak{g} \rightarrow \operatorname{Hom}\left(J^{1} E, E\right)
$$

one writes $\nabla_{X} \sigma:=\nabla(X)\left(J^{1} \sigma\right)(X \in \Gamma(\mathfrak{g}), \sigma \in \Gamma(E))$. The following elementary result characterizes $\mathfrak{g}$-connections as certain differential operators, and shows that $\mathfrak{g}$-representations are just flat $\mathfrak{g}$-connections, where flatness takes a familiar form. 


\section{Proposition.}

(1) $\nabla: \mathfrak{g} \rightarrow \operatorname{Hom}\left(J^{1} E, E\right)$ is a $\mathfrak{g}$-connection if and only if

$$
\nabla_{X}(f \sigma)=f \nabla_{X} \sigma+d f(\# X) \sigma
$$

for all smooth functions $f$ on $M$ and sections $X \in \Gamma(\mathfrak{g}), \sigma \in \Gamma(E)$.

(2) In that case $\nabla: \mathfrak{g} \rightarrow \mathfrak{g l}(E)$ is a $\mathfrak{g}$-representation if and only if its curvature, in the sense of Sect.4.1, vanishes. This curvature is a section of $\operatorname{Alt}^{2}(\mathfrak{g}, \operatorname{Hom}(E, E))$ and satisfies

$$
\operatorname{curv} \nabla(X, Y) \sigma=\left(\nabla_{X} \nabla_{Y}-\nabla_{Y} \nabla_{X}-\nabla_{[X, Y]}\right) \sigma .
$$

4.5. $T M$-connections. A $T M$-connection is simply an affine connection in the usual sense. Given a $T M$-connection $\nabla: T M \rightarrow \mathfrak{g l}(E)$ on $E$, and a section $\sigma$ of $E$, we write $\nabla \cdot \sigma$ for the section of $T^{*} M \otimes E \cong \operatorname{Hom}(T M, E)$ defined by

$$
(\nabla \cdot \sigma) V:=\nabla_{V} \sigma=\nabla(V)\left(J^{1} \sigma\right), \quad V \in \Gamma(T M) .
$$

Then $\nabla$ determines a splitting $J^{1} E \stackrel{s}{\leftarrow} E$ of the exact sequence 4.2(1), given by

$$
s \sigma=J^{1} \sigma-\nabla \cdot \sigma, \quad \sigma \in \Gamma(E) .
$$

Conversely, (1) implicitly defines a $T M$-connection $\nabla$ for every such splitting $s$.

Remarks.

(2) The preceding observations are transparent if one observes that prolongation $\Gamma\left(J^{1} E\right) \stackrel{J^{1}}{\longleftarrow} \Gamma(E)$ is a god-given splitting for the sequence obtained by applying $\Gamma(\cdot)$ to 4.2(1):

$$
0 \rightarrow \Gamma\left(T^{*} M \otimes E\right) \hookrightarrow \Gamma\left(J^{1} E\right) \rightarrow \Gamma(E) \rightarrow 0 .
$$

(3) Suppose $M$ is simply connected. Then a representation of $T M$ is just a vector bundle $E$, together with a trivialization $E \cong E_{0} \times M$. Indeed, suppose $\nabla: T M \rightarrow \mathfrak{g l}(E)$ is such a representation (i.e., a flat $T M$-connection on $E)$. By the groupoid version of Lie's Second Fundamental Theorem (see, e.g., 5), the Lie algebroid morphism $\nabla$ lifts to a groupoid morphism $\Phi: M \times M \rightarrow \mathrm{GL}(E)$. If $m_{0} \in M$ is fixed and $E_{0}$ is the fiber over $m_{0}$, the trivialization is given by

$$
\begin{gathered}
E_{0} \times M \stackrel{\sim}{\longrightarrow} E, \\
(\xi, m) \mapsto \Phi\left(m_{0}, m\right) \xi .
\end{gathered}
$$

More invariantly, one identifies $E_{0}$ with the space of $\nabla$-parallel sections of $E$.

4.6. Dual connections and torsion. Let $\mathfrak{g}$ be a Lie algebroid and $\nabla$ a $\mathfrak{g}$-connection on itself. Generalizing Sect. 3.1, we define the dual of $\nabla$ to be the $\mathfrak{g}$-connection $\nabla^{*}$ on $\mathfrak{g}$ defined by

$$
\nabla_{X}^{*} Y:=\nabla_{Y} X+[X, Y]_{\mathfrak{g}} .
$$

One has 'duality' in the sense that $\nabla^{* *}=\nabla$.

The torsion of $\nabla$ is the section, tor $\nabla$, of $\operatorname{Alt}^{2}(\mathfrak{g}, \mathfrak{g})$ measuring the difference between $\nabla$ and its dual:

$$
\text { tor } \nabla(X, Y):=\nabla_{X} Y-\nabla_{X}^{*} Y=\nabla_{X} Y-\nabla_{Y} X-[X, Y] \text {. }
$$

The torsion or curvature of $\nabla$ can be expressed in terms of the torsion and curvature of $\nabla^{*}$ (and, by duality, vice versa). 
Proposition. Let $\nabla$ be a $\mathfrak{g}$-connection on $\mathfrak{g}$, and $\nabla^{*}$ its dual. Then:

$$
\begin{aligned}
& \operatorname{tor} \nabla=-\operatorname{tor} \nabla^{*}, \\
& \left\{\begin{aligned}
\operatorname{curv} \nabla(X, Y) Z= & \left(\nabla_{Z}^{*} \operatorname{tor} \nabla^{*}\right)(X, Y)+\operatorname{curv} \nabla^{*}(X, Z) Y \\
+ & \operatorname{curv} \nabla^{*}(Z, Y) X ; \quad X, Y, Z \subset \mathfrak{g} .
\end{aligned}\right.
\end{aligned}
$$

In particular:

(3) If $\nabla^{*}$ is flat, then $\nabla$ is flat if and only if tor $\nabla^{*}$ is $\nabla^{*}$-parallel.

\section{Cartan connections}

In Sect.2.3 a Cartan connection on a Lie algebroid $\mathfrak{g}$ was defined as a $T M$ connection on $\mathfrak{g}$ 'compatible' with the bracket on $\mathfrak{g}$. Pivotal to understanding the compatibility condition is the observation that the first jet bundle $J^{1} \mathfrak{g}$ is also a Lie algebroid, a fact noted in [11. While the bracket on a Lie algebra $\mathfrak{g}$ determines a canonical representation ad: $\mathfrak{g} \rightarrow \mathfrak{g l}(\mathfrak{g})$, the adjoint representation, the analogue for a Lie algebroid $\mathfrak{g}$ is a representation ad: $J^{1} \mathfrak{g} \rightarrow \mathfrak{g l}(\mathfrak{g})$ defined by $\operatorname{ad}_{J^{1} X} Y:=[X, Y]_{\mathfrak{g}}$. We show that a Cartan connection $\nabla$ on $\mathfrak{g}$ is compatible with the bracket on $\mathfrak{g}$ when the associated splitting $J^{1} \mathfrak{g} \stackrel{s}{\longleftarrow} \mathfrak{g}$ of

$$
0 \rightarrow T^{*} M \otimes \mathfrak{g} \hookrightarrow J^{1} \mathfrak{g} \rightarrow \mathfrak{g} \rightarrow 0
$$

is a Lie algebroid morphism. In that case, the composite

$$
\mathfrak{g} \stackrel{s}{\rightarrow} J^{1} \mathfrak{g} \stackrel{\text { ad }}{\longrightarrow} \mathfrak{g l}(\mathfrak{g})
$$

delivers a representation of $\mathfrak{g}$ on itself. There is moreover, a representation of $\mathfrak{g}$ on $T M$, with respect to which the anchor $\#: \mathfrak{g} \rightarrow T M$ is equivariant. Details now follow.

5.1. The first jet of a Lie algebroid. If $\mathfrak{g}$ is the Lie algebroid of a Lie groupoid $G$, then $J^{1} \mathfrak{g}$ is a model for the Lie algebroid of $J^{1} G$, the groupoid of one-jets of bisections of $G$. This suggests a definition for the bracket on $J^{1} \mathfrak{g}$ more generally, and this definition agrees with the one to be described below. See also [6].

Lemma. For any Lie algebroid $\mathfrak{g}$ over $M$, the formula

$$
\kappa_{X}(\alpha \otimes Y):=\mathcal{L}_{\# X} \alpha \otimes Y+\alpha \otimes[X, Y]_{\mathfrak{g}}
$$

defines a Lie algebra representation,

$$
X \mapsto \kappa_{X}: \Gamma(\mathfrak{g}) \rightarrow \mathfrak{g l}\left(\Gamma\left(T^{*} M \otimes \mathfrak{g}\right)\right) .
$$

Viewing sections of $T^{*} M \otimes \mathfrak{g}$ as sections of $\operatorname{Hom}(T M, \mathfrak{g})$, one has

$$
\left(\kappa_{X} \phi\right) V:=[X, \phi(V)]_{\mathfrak{g}}+\phi\left([V, \# X]_{T M}\right) .
$$

In making $J^{1} \mathfrak{g}$ a Lie algebroid, the inevitable choice of anchor is the composite $J^{1} \mathfrak{g} \rightarrow \mathfrak{g} \stackrel{\#}{\longrightarrow} T M$, which we again denote by \#. A Lie algebroid bracket on $J^{1} \mathfrak{g}$ is a Lie bracket on the space of sections $\Gamma\left(J^{1} \mathfrak{g}\right)$, which, by Remark 4.5)(2), is isomorphic to $\Gamma(\mathfrak{g}) \oplus \Gamma\left(T^{*} M \otimes \mathfrak{g}\right)$. A natural choice of bracket is one making $\Gamma\left(J^{1} \mathfrak{g}\right)$ a semidirect product of $\Gamma(\mathfrak{g})$ and $\Gamma\left(T^{*} M \otimes \mathfrak{g}\right)$.

First, we make $T^{*} M \otimes \mathfrak{g}$ a bundle of Lie algebras by defining

$$
\left[\phi_{1}, \phi_{2}\right]_{T^{*} M \otimes \mathfrak{g}}:=\phi_{2} \circ \# \circ \phi_{1}-\phi_{1} \circ \# \circ \phi_{2} .
$$


Next we use the representation $\kappa$ above to make $\Gamma\left(J^{1} \mathfrak{g}\right)$ a semidirect product of the Lie algebras $\Gamma(\mathfrak{g})$ and $\Gamma\left(T^{*} M \otimes \mathfrak{g}\right)$. That is, we define

$$
\begin{aligned}
{\left[J^{1} X_{1}+\phi_{1}, J^{1} X_{2}+\phi_{2}\right]_{J^{1} \mathfrak{g}} } & :=J^{1}\left[X_{1}, X_{2}\right]_{\mathfrak{g}}+\left[\phi_{1}, \phi_{2}\right]_{T^{*} M \otimes \mathfrak{g}} \\
& +\kappa_{X_{1}} \phi_{2}-\kappa_{X_{2}} \phi_{1} .
\end{aligned}
$$

In formulas (2) and (3) $, \phi_{1}, \phi_{2} \in \Gamma\left(T^{*} M \otimes \mathfrak{g}\right)$ and $X_{1}, X_{2} \in \Gamma(\mathfrak{g})$ are arbitrary sections. The compositions in (2) make sense because we identify sections of $T^{*} M \otimes$ $\mathfrak{g}$ with sections of $\operatorname{Hom}(T M, \mathfrak{g})$.

With the above choice of anchor and bracket, $J^{1} \mathfrak{g}$ becomes a Lie algebroid, containing $T^{*} M \otimes \mathfrak{g}$ as a subalgebroid.

Our definition of the Lie algebroid structure of $J^{1} \mathfrak{g}$ is natural in a categorical sense: Suppose that $f: E \rightarrow F$ is a morphism of vector bundles over $M$, covering the identity. Then one defines $J^{1} f: J^{1} E \rightarrow J^{1} F$ by $J^{1} f\left([\sigma]_{m}^{1}\right):=J^{1}\left([f \sigma]_{m}^{1}\right)$ and obtains the following commutative diagram:

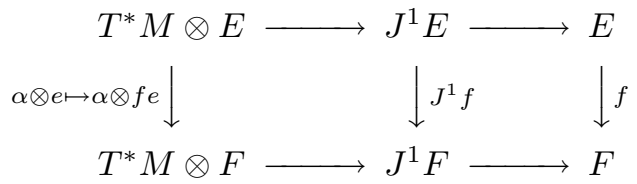

Proposition. If $f: \mathfrak{g} \rightarrow \mathfrak{h}$ is a morphism of Lie algebroids, then so is $J^{1} f: J^{1} \mathfrak{g} \rightarrow$ $J^{1} \mathfrak{h}$.

5.2. Adjoint representations. The Lie algebroid structure of $J^{1} \mathfrak{g}$ fixed above guarantees the following result.

Proposition. For any Lie algebroid $\mathfrak{g}$, the formula

$$
\operatorname{ad}_{J^{1} X} Y:=[X, Y]_{\mathfrak{g}}, \quad X, Y \in \Gamma(\mathfrak{g}),
$$

defines a representation ad of $J^{1} \mathfrak{g}$ on $\mathfrak{g}$ that we call the adjoint representation. If $\operatorname{ad}^{\prime}: T^{*} M \otimes \mathfrak{g} \rightarrow \operatorname{Hom}(\mathfrak{g}, \mathfrak{g})$ is defined by

$$
\operatorname{ad}^{\prime}(\alpha \otimes x) y:=-\alpha(\# y) x,
$$

then the following is a commutative diagram of Lie algebroid morphisms with exact rows:

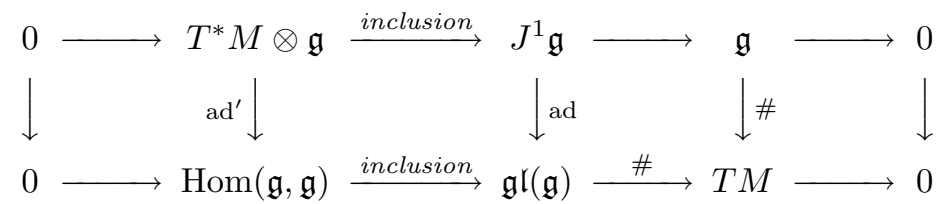

For any smooth manifold $M$, the adjoint representation ad: $J^{1}(T M) \rightarrow \mathfrak{g l}(T M)$ is a Lie algebroid isomorphism.

Caution. Note the minus sign in (1), necessary for diagram commutativity. Note also the differing order of terms in definition [5.1(2) of $\left[\phi_{1}, \phi_{2}\right]_{T^{*} M \otimes \mathfrak{g}}$, compared with that for $\left[\phi_{1}, \phi_{2}\right]_{\operatorname{Hom}(\mathfrak{g}, \mathfrak{g})}$ in $4.2(2)$ (taking $\left.E=\mathfrak{g}\right) \mathbf{1}$

\footnotetext{
${ }^{1}$ For this reason, in the special case $\mathfrak{g}=T M$, one must understand the canonical identification $T^{*} M \otimes T M \cong \operatorname{Hom}(T M, T M)$ as an antihomomorphism of Lie algebroids. This sign annoyance can only be shifted, not eliminated.
} 
5.3. Bracket compatibility. Let $\nabla$ denote an arbitrary $T M$-connection on a Lie algebroid $\mathfrak{g}$, let $J^{1} \mathfrak{g} \stackrel{s}{\leftarrow} \mathfrak{g}$ denote the corresponding splitting of $0 \rightarrow T^{*} M \otimes \mathfrak{g} \hookrightarrow$ $J^{1} \mathfrak{g} \rightarrow \mathfrak{g} \rightarrow 0$ (described in Sect.4.5), and consider the composite morphisms

$$
\begin{array}{r}
\mathfrak{g} \stackrel{s}{\longrightarrow} J^{1} \mathfrak{g} \stackrel{\mathrm{ad}}{\longrightarrow} \mathfrak{g l}(\mathfrak{g}), \\
\mathfrak{g} \stackrel{s}{\longrightarrow} J^{1} \mathfrak{g} \stackrel{J^{1} \#}{\longrightarrow} J^{1}(T M) \stackrel{\text { ad }}{\longrightarrow} \mathfrak{g l}(T M) .
\end{array}
$$

These define, respectively, a $\mathfrak{g}$-connection on $\mathfrak{g}$ and a $\mathfrak{g}$-connection on $T M$. Both connections will be denoted $\nabla$. Using 4.5(1) and diagram [5.1(4), one computes for $X \in \Gamma(\mathfrak{g})$,

$$
\begin{array}{r}
\bar{\nabla}_{X} Y=\nabla_{\# Y} X+[X, Y]_{\mathfrak{g}}, \quad Y \in \Gamma(\mathfrak{g}), \\
\bar{\nabla}_{X} V=\# \nabla_{V} X+[\# X, V]_{T M}, \quad V \in \Gamma(T M) .
\end{array}
$$

With respect to these connections, the anchor $\#: \mathfrak{g} \rightarrow T M$ is connection-preserving:

$$
\# \bar{\nabla}_{X} Y=\bar{\nabla}_{X} \# Y, \quad X, Y \in \Gamma(\mathfrak{g}) .
$$

Proposition. The connection $\nabla$ is compatible with the bracket on $\mathfrak{g}$ in the sense of Definition 2.3 (i.e., is a Cartan connection) if and only if the corresponding splitting $J^{1} \mathfrak{g} \stackrel{s}{\leftarrow} \mathfrak{g}$ above is a Lie algebroid morphism. In that case, the $\mathfrak{g}$-connections on $\mathfrak{g}$ and $T M$ given by (3) and (4) are representations.

Proof. With the assistance of 4.5(1) and 5.1(3), we compute

$$
\begin{aligned}
\operatorname{curv} s(X, Y)= & {[s X, s Y]_{J^{1} \mathfrak{g}}-s[X, Y]_{\mathfrak{g}} } \\
= & {\left[J^{1} X, J^{1} Y\right]_{J^{1} \mathfrak{g}}-\left[J^{1} X, \nabla \cdot Y\right]_{J^{1} \mathfrak{g}}-\left[\nabla \cdot X, J^{1} Y\right]_{J^{1} \mathfrak{g}} } \\
& +[\nabla \cdot X, \nabla \cdot Y]_{J^{1} \mathfrak{g}}-J^{1}[X, Y]_{\mathfrak{g}}+\nabla \cdot[X, Y]_{\mathfrak{g}} \\
= & -\kappa_{X}(\nabla \cdot Y)+\kappa_{Y}(\nabla \cdot X) \\
& +(\nabla \cdot Y) \circ \# \circ(\nabla \cdot X)-(\nabla \cdot X) \circ \# \circ(\nabla \cdot Y) \\
& +\nabla \cdot[X, Y]_{\mathfrak{g}} .
\end{aligned}
$$

Applying [5.1(1), we obtain

$$
\begin{aligned}
\operatorname{curv} s(X, Y) V= & -\left[X, \nabla_{V} Y\right]_{\mathfrak{g}}-\nabla_{[V, \# X]_{T M}} Y+\left[Y, \nabla_{V} X\right]_{\mathfrak{g}}+\nabla_{[V, \# Y]_{T M}} X \\
& +\nabla_{\# \nabla_{V} X} Y-\nabla_{\# \nabla_{V} Y} X+\nabla_{V}[X, Y]_{\mathfrak{g}} \\
= & \nabla_{V}[X, Y]_{\mathfrak{g}}-\left[\nabla_{V} X, Y\right]_{\mathfrak{g}}-\left[X, \nabla_{V} Y\right]_{\mathfrak{g}} \\
& -\nabla_{\bar{\nabla}_{Y} V} X+\nabla_{\bar{\nabla}_{X} V} Y, \quad \text { by (44). }
\end{aligned}
$$

Thus curv $s=0$ if and only if 2.3(1) holds, which proves the first claim. The second claim holds because the morphisms in (11) and (2) are Lie algebroid morphisms whenever $s: \mathfrak{g} \rightarrow J^{1} \mathfrak{g}$ is a Lie algebroid morphism. (The other morphisms appearing in (11) and (2) are Lie algebroid morphisms by Propositions 5.1 and Proposition 5.2.)

5.4. On the curvature of Cartan connections. Let $\nabla$ be a Cartan connection. Then

$$
\operatorname{curv} \nabla(\# X, \# Y) Z=\left(\bar{\nabla}_{Z} \text { tor } \bar{\nabla}\right)(X, Y), \quad X, Y, Z \in \Gamma(\mathfrak{g}),
$$

where $\bar{\nabla}$ denotes the representation of $\mathfrak{g}$ on itself determined by $\nabla$. 
Proof of (11). From [5.3(3) follows the identity $\nabla_{\# X} Y=\bar{\nabla}_{X}^{*} Y$, where $\bar{\nabla}^{*}$ is the dual of $\bar{\nabla}: \mathfrak{g} \rightarrow \mathfrak{g l}(\mathfrak{g})$ (see Sect.4.6). So

$$
\begin{aligned}
\operatorname{curv} \nabla(\# X, \# Y) Z & =\operatorname{curv} \bar{\nabla}^{*}(X, Y) Z \\
& =\left(\bar{\nabla}_{Z} \text { tor } \bar{\nabla}\right)(X, Y),
\end{aligned}
$$

by Proposition 4.6(2) and the flatness of $\bar{\nabla}$.

Combining (11) with Theorem A, we have:

Corollary. A transitive Cartan algebroid $(\mathfrak{g}, \nabla)$ is locally symmetric if and only if tor $\bar{\nabla}$ is $\bar{\nabla}$-parallel, where $\bar{\nabla}$ denotes the corresponding representation of $\mathfrak{g}$ on itself.

The transitive case is discussed further in Sect.6.

5.5. Invariant differential operators. Let $(\mathfrak{g}, \nabla)$ be a Cartan algebroid on $M$. Let a $\mathfrak{g}$-tensor on $M$ be any section of some representation $E$ of $\mathfrak{g}$. As $\nabla$ determines representations of $\mathfrak{g}$ on $T M$ and itself, all differential forms and all differential $\mathfrak{g}$ forms (sections of $\mathrm{Alt}^{k}(\mathfrak{g})$, for some $k$ ) are $\mathfrak{g}$-tensors.

There is a fundamental operator $D$ acting on $\mathfrak{g}$-tensors: If $\tau$ is a section of $E$ and $\rho: \mathfrak{g} \rightarrow \mathfrak{g l}(E)$ is a representation, then $D \tau \in \Gamma\left(\mathfrak{g}^{*} \otimes E\right)$ is defined by

$$
\langle D \tau, X\rangle=\rho_{X} \tau, \quad X \in \Gamma(\mathfrak{g}) .
$$

Since $D \tau$ is another $\mathfrak{g}$-tensor, the fundamental operator $D$ can be iterated to obtain higher order differential operators.

As is well known, there is an exterior derivative operator $d$ acting on $E$-valued $\mathfrak{g}$-forms, whenever $(E, \rho)$ is a representation of $\mathfrak{g}$ :

$$
\begin{gathered}
d: \Gamma\left(\operatorname{Alt}^{k}(\mathfrak{g}, E)\right) \rightarrow \Gamma\left(\operatorname{Alt}^{k+1}(\mathfrak{g}, E)\right), \\
d \theta\left(X_{0}, \ldots, X_{k}\right):=\sum_{i=0}^{k}(-1)^{i} \rho_{X_{k}}\left(\theta\left(X_{0}, \ldots, \hat{X}_{i}, \ldots, X_{k}\right)\right) \\
-\sum_{i<j}(-1)^{i+j+1} \theta\left(\left[X_{i}, X_{j}\right]_{\mathfrak{g}}, X_{0}, \ldots, \hat{X}_{i}, \ldots, \hat{X}_{j}, \ldots, X_{k}\right) .
\end{gathered}
$$

One has $d^{2}=0$, following from the fact that $\operatorname{curv} \rho=0$.

Let $\omega \in \Gamma\left(\operatorname{Alt}^{1}(\mathfrak{g}, \mathfrak{g})\right)$ denote the tautological form, $\omega(X)=X$. Then $D \omega=0$ and $d \omega=\operatorname{tor} \bar{\nabla}$, where $\bar{\nabla}$ is the representation of $\mathfrak{g}$ on itself induced by $\nabla$. The exterior derivative $d$ can be expressed in terms of the fundamental operator $D$ according to

$$
d \theta=\omega \wedge D \theta+\theta^{d \omega} .
$$

Here we view $D \theta$ as a $\mathfrak{g}^{*} \otimes E$-valued $\mathfrak{g}$-form, and the wedge implies a contraction $\mathfrak{g} \otimes\left(\mathfrak{g}^{*} \otimes E\right) \rightarrow E$. Also,

$$
\theta^{d \omega}\left(X_{0}, \ldots, X_{k}\right):=\sum_{i<j}(-1)^{i+j+1} \theta\left(d \omega\left(X_{i}, X_{j}\right), X_{0}, \ldots, \hat{X}_{i}, \ldots, \hat{X}_{j}, \ldots, X_{k}\right),
$$

unless $k=0$, in which case $\theta^{d \omega}:=0$. 


\section{Transitive Cartan algebroids}

The remainder of the paper concerns transitive Cartan algebroids.

6.1. Cartan connections recharacterized. As we have observed above, every Cartan connection $\nabla$ on a Lie algebroid $\mathfrak{g}$ determines a representation of $\mathfrak{g}$ on itself. In the transitive case the Cartan connection is uniquely determined by this representation, as we now explain.

Let $\mathfrak{g}$ be a transitive Lie algebroid and let $\mathfrak{h} \subset \mathfrak{g}$ denote the kernel of its anchor. So we have an exact sequence

$$
0 \rightarrow \mathfrak{h} \hookrightarrow \mathfrak{g} \stackrel{\#}{\longrightarrow} T M \rightarrow 0
$$

The bracket on $\mathfrak{g}$ determines a canonical representation $\rho$ of $\mathfrak{g}$ on $\mathfrak{h}$ :

$$
\rho_{X} Y=[X, Y]_{\mathfrak{g}}, \quad X \in \Gamma(\mathfrak{g}), Y \in \Gamma(\mathfrak{h}) .
$$

If $\nabla$ is a Cartan connection on $\mathfrak{g}$, the corresponding representation $\bar{\nabla}$ of $\mathfrak{g}$ on itself is given by

$$
\bar{\nabla}_{X} Y:=\nabla_{\# Y} X+[X, Y]_{\mathfrak{g}}
$$

This representation leaves the subbundle $\mathfrak{h} \subset \mathfrak{g}$ invariant, and its restriction to $\mathfrak{h}$ is just the canonical representation $\rho$ above. According to (1),

$$
\nabla_{V} X=\bar{\nabla}_{X} Y+[Y, X]_{\mathfrak{g}} \quad \text { whenever } \quad \# \mathrm{Y}=\mathrm{V} \text {. }
$$

The following result is readily verified:

Proposition. Let $\mathfrak{g}$ be a transitive Lie algebroid with kernel $\mathfrak{h}$. Let $\mathfrak{g} \stackrel{t}{\leftarrow} T M$ be an arbitrary vector bundle splitting of the exact sequence

$$
0 \rightarrow \mathfrak{h} \rightarrow \mathfrak{g} \stackrel{\#}{\rightarrow} T M \rightarrow 0
$$

Then for each representation $D$ of $\mathfrak{g}$ on itself extending the canonical representation $\rho$ of $\mathfrak{g}$ on $\mathfrak{h}$, the $T M$-connection $\nabla$ on $\mathfrak{g}$, defined by

$$
\nabla_{V} X=D_{X} t V+[t V, X]_{\mathfrak{g}}
$$

is independent of the choice of splitting $t$, and is a Cartan connection satisfying $\bar{\nabla}=D$. In particular, the map $\nabla \mapsto \bar{\nabla}$ is a bijection from the set of Cartan connections on $\mathfrak{g}$ to the set of representations of $\mathfrak{g}$ on itself extending $\rho$.

Given an arbitrary splitting $\mathfrak{g} \stackrel{t}{\leftarrow} T M$, we can also write 5.4(1) as

$$
\operatorname{curv} \nabla(V, W) Z=\left(\bar{\nabla}_{Z} \text { tor } \bar{\nabla}\right)(t V, t W), \quad V, W \in \Gamma(T M) .
$$

6.2. Reductive connections. A Cartan connection $\nabla$ on $\mathfrak{g}$ will be called reductive if the corresponding representation $\bar{\nabla}$ of $\mathfrak{g}$ on itself splits over the exact sequence $\mathfrak{h} \rightarrow \mathfrak{g} \stackrel{\#}{\longrightarrow} T M$. That is, if there exists a splitting $\mathfrak{g} \stackrel{t}{\leftarrow} T M$ such that the induced isomorphism $\mathfrak{g} \cong T M \oplus \mathfrak{h}$ of vector bundles is an isomorphism of $\mathfrak{g}$-representations; it suffices to check that $t$ is equivariant, i.e., that

$$
\bar{\nabla}_{X} t V=t \bar{\nabla}_{X} V, \quad X \in \Gamma(\mathfrak{g}), V \in \Gamma(T M)
$$

where $\bar{\nabla}$ on the left refers to the representation of $\mathfrak{g}$ on itself determined by $\nabla$, while on the right it refers to that on $T M$ (see Sect.5.3). One can express the Cartan connection in terms of $t$ and $\bar{\nabla}$ :

$$
\nabla_{V} X=t \bar{\nabla}_{X} V+[t V, X]_{\mathfrak{g}}
$$


Conversely, for any splitting $t$ and representation $\bar{\nabla}$ of $\mathfrak{g}$ on $T M$, (2) defines a reductive Cartan connection.

6.3. Infinitesimal $G$-structures. A $G$-structure on a manifold $M$ is a reduction of its general linear frame bundle; see [12. The corresponding groupoid notion is a (transitive) subgroupoid of the frame groupoid $\mathrm{GL}(T M)$, frame groupoids having been defined in Sect.4.2. Noting that the adjoint representation ad: $J^{1}(T M) \rightarrow$ $\mathfrak{g l}(T M)$ is an isomorphism, we define an infinitesimal $G$-structure to be any Lie subalgebroid of $J^{1}(T M) 2$ If $\mathfrak{g} \subset J^{1}(T M)$ is an infinitesimal $G$-structure, then the restriction ad: $\mathfrak{g} \rightarrow \mathfrak{g l}(T M)$ of the adjoint representation (see Sect.5.2) is a canonical representation of $\mathfrak{g}$ on $T M$. It is natural to restrict attention to Cartan connections $\nabla$ on $\mathfrak{g}$ for which the corresponding representation $\bar{\nabla}$ of $\mathfrak{g}$ on $T M$ is this restricted adjoint representation.

The following generalizes Corollary 3.3 .

Theorem B. Consider a transitive infinitesimal G-structure $\mathfrak{g} \subset J^{1}(T M)$ and let $\mathfrak{h} \subset T^{*} M \otimes T M$ denote the kernel of its anchor. Let $\nabla$ be a reductive Cartan connection on $\mathfrak{g}$ with $\bar{\nabla}=\mathrm{ad}$, and let $\mathfrak{g} \stackrel{t}{\leftarrow} T M$ denote a corresponding splitting of $0 \rightarrow \mathfrak{h} \hookrightarrow \mathfrak{g} \rightarrow T M \rightarrow 0$ (i.e., 6.2(2) holds). Let $\nabla^{\mathrm{L}-\mathrm{C}}$ denote the composite

$$
T M \stackrel{t}{\rightarrow} \mathfrak{g} \stackrel{\text { ad }}{\longrightarrow} \mathfrak{g l}(T M),
$$

which is a TM-connection on TM. Then the Cartan algebroid $(\mathfrak{g}, \nabla)$ is locally symmetric if and only if tor $\nabla^{\mathrm{L}-\mathrm{C}}$ and curv $\nabla^{\mathrm{L}-\mathrm{C}}$ are both $\mathfrak{h}$-invariant and $\nabla^{\mathrm{L}-\mathrm{C}}-$ parallel.

Proof. The curvature of $\nabla$ is given by 6.1(2). With the help of the equivariance property 6.2(1), one easily rewrites this as

$$
\operatorname{curv} \nabla(V, W) Z=\bar{\nabla}_{Z}\left(t^{*} \text { tor } \bar{\nabla}\right)(V, W)
$$

where

$$
t^{*} \operatorname{tor} \bar{\nabla}(V, W):=\text { tor } \bar{\nabla}(t V, t W) .
$$

Appealing to equivariance and the definition of torsion, one obtains

$$
\begin{aligned}
t^{*} \text { tor } \bar{\nabla}(V, W) & =t \bar{\nabla}_{t V} W-t \bar{\nabla}_{t W} V-[t V, t W]_{\mathfrak{g}} \\
& =t\left(\operatorname{ad}_{t V} W-\operatorname{ad}_{t W} V-[V, W]_{T M}\right)-\operatorname{curv} t(V, W) \\
& =t\left(\operatorname{tor} \nabla^{\mathrm{L}-\mathrm{C}}(V, W)\right)-\operatorname{curv} t(V, W) .
\end{aligned}
$$

We claim that

$$
\operatorname{curv} t(V, W)=-\operatorname{curv} \nabla^{\mathrm{L}-\mathrm{C}}(V, W)
$$

Indeed, we have

$$
\begin{aligned}
\operatorname{curv} \nabla^{\mathrm{L}-\mathrm{C}}(V, W) & =\operatorname{curv}(\operatorname{ad} \circ t)(V, W) \\
& =\left([\operatorname{ad}(t V), \operatorname{ad}(t W)]_{\mathfrak{g r}(T M)}-\operatorname{ad}\left(t[V, W]_{T M}\right)\right) \\
& =\operatorname{ad}\left([t V, t W]_{\mathfrak{g}}-t[V, W]_{T M}\right) \\
& =\operatorname{ad}^{\prime}(\operatorname{curv} t(V, W)),
\end{aligned}
$$

implying (see 5.2(1)) that

$$
\operatorname{curv} \nabla^{\mathrm{L}-\mathrm{C}}(V, W) U=-\operatorname{curv} t(V, W) U .
$$

\footnotetext{
${ }^{2}$ These are a special case of the infinitesimal geometric structures defined in [2].
} 
Substituting (3) into (2), we obtain

$$
t^{*} \operatorname{tor} \bar{\nabla}(V, W)=t\left(\operatorname{tor} \nabla^{\mathrm{L}-\mathrm{C}}(V, W)\right)+\operatorname{curv} \nabla^{\mathrm{L}-\mathrm{C}}(V, W) .
$$

Using this and equivariance in (1), we arrive at the formula

$$
\operatorname{curv} \nabla(V, W) Z=t\left(\left(\bar{\nabla}_{Z} \operatorname{tor} \nabla^{\mathrm{L}-\mathrm{C}}\right)(V, W)\right)+\left(\bar{\nabla}_{Z} \operatorname{curv} \nabla^{\mathrm{L}-\mathrm{C}}\right)(V, W) .
$$

The first term on the right-hand side is a section of $t(T M) \subset \mathfrak{g}$, while the second term is a section of $\mathfrak{h}$. So curv $\nabla=0$ if and only if tor $\nabla^{\mathrm{L}-\mathrm{C}}$ and curv $\nabla^{\mathrm{L}-\mathrm{C}}$ are both $\bar{\nabla}$-parallel. Now for any vector field $V$ on $M$, we have

$$
\begin{aligned}
\bar{\nabla}_{Z} V & =Z(V) \quad \text { for } \quad Z \in \Gamma(\mathfrak{h}) \subset \Gamma\left(T^{*} M \otimes T M\right), \\
\text { while } \quad \bar{\nabla}_{t W} V & =\nabla_{W}^{\mathrm{L}-\mathrm{C}} V \quad \text { for } \quad W \in \Gamma(T M) .
\end{aligned}
$$

So being $\bar{\nabla}$-parallel is the same as being simultaneously $\mathfrak{h}$-invariant and $\nabla^{\mathrm{L}-\mathrm{C}_{-}}$ parallel. The conclusion of the theorem is now a consequence of Theorem A.

\section{Cartan GeOmetries}

Let $G_{0}$ be a Lie group and $H_{0}$ a closed subgroup. Let $\mathfrak{g}_{0}$ and $\mathfrak{h}_{0}$ denote the Lie algebras of these groups. Then a Cartan geometry modeled on $G_{0} / H_{0}$ is a smooth manifold $M$, together with a principal $H_{0}$-bundle $\pi: P \rightarrow M$, and an absolute parallelism $\omega \in \Omega^{1}\left(P, \mathfrak{g}_{0}\right)$ of $P$. One requires that $\omega$ is $H_{0}$-equivariant, where $H_{0}$ acts on $\mathfrak{g}_{0}$ via the adjoint action. We suppose $H_{0}$ acts on $P$ from the right. For details, see 16 .

A one-form $\omega$ as above is known as a Cartan connection, but to distinguish it from the connections defined in Sect.2.3. we will call $\omega$ a classical Cartan connection.

7.1. Curvature. The curvature of the Cartan geometry is the $\mathfrak{g}_{0}$-valued two-form on $P$ defined by

$$
\Omega:=d \omega+\frac{1}{2}[\omega, \omega]_{\mathfrak{g}_{0}} .
$$

The model $G_{0} / H_{0}$ is itself a Cartan geometry (take $M=G_{0} / H_{0}, P=G_{0}$, and let $\omega$ be the left-invariant Mauer-Cartan form on $G_{0}$ ), and in this case the curvature vanishes. Conversely, if $M$ is an arbitrary Cartan geometry modeled on $G_{0} / H_{0}$, then $\Omega=0$ implies that $M$ is isomorphic to $G_{0} / H_{0}$, assuming certain global obstructions also vanish [16, Theorem 5.3]. So curvature is the local measure of deviation from the prescribed model.

We now describe a canonical, transitive, Cartan algebroid $(\mathfrak{g}, \nabla)$ associated with a Cartan geometry $(M, P \stackrel{\pi}{\rightarrow} M, \omega)$ modelled on $G_{0} / H_{0}$.

7.2. The Lie algebroid $\mathfrak{g}$. The Lie algebroid is given by $\mathfrak{g}:=(T P) / H_{0}$, its anchor being the map \#: $\mathfrak{g} \rightarrow T M$ sending $v \bmod H_{0}$ to $T \pi \cdot v$. Sections of $\mathfrak{g}$ are in one-toone correspondence with the $H_{0}$-invariant vector fields on $P$. Since the collection of such vector fields is closed under the Jacobi-Lie bracket $[\cdot, \cdot]_{T P}$, we obtain a Lie bracket $[\cdot, \cdot]_{\mathfrak{g}}$ on $\Gamma(\mathfrak{g})$. This bracket makes $\mathfrak{g}$ into a Lie algebroid.

An arbitrary vector field $X$ on $P$ is $H_{0^{-}}$-invariant if and only if $\omega(X)$ is $H_{0^{-}}$ equivariant. 
7.3. The Cartan connection $\nabla$ on $\mathfrak{g}$. As an absolute parallelism, the classical Cartan connection $\omega$ determines a flat affine connection $D$ on $T P$. Implicitly, $D$ is defined by

$$
\omega\left(D_{X} Y\right)=\mathcal{L}_{X}(\omega(Y)), \quad X, Y \in \Gamma(P),
$$

where $\mathcal{L}$ is a Lie derivative. On the other hand, if $X$ and $Y$ are both $H_{0}$-invariant, then so is $D_{X} Y$, which shows that $D$ may also be viewed as a flat $\mathfrak{g}$-connection on $\mathfrak{g}$, i.e., as a representation of $\mathfrak{g}$ on itself. By Proposition 6.1, $D$ determines a unique Cartan connection $\nabla$ on $\mathfrak{g}$ satisfying $\bar{\nabla}=D$.

The Cartan algebroid $(\mathfrak{g}, \nabla)$ above encodes all local information concerning the Cartan geometry, with the exception of certain model information. One can recover the group $H_{0}$, up to cover, but at best reconstructs $\mathfrak{g}_{0}$ as a representation of $H_{0}$; unless $\nabla$ is flat (see Theorem $\mathrm{C}$ below), the Lie algebra structure of $\mathfrak{g}_{0}$ is lost. We suppress details.

7.4. Local symmetry. For convenience, we now identify the $\mathfrak{g}_{0}$-valued two-forms $d \omega,[\omega, \omega]_{\mathfrak{g}_{0}}$ and $\Omega$ with $T P$-valued two-forms on $P$. This we may do using the absolute parallelism $\omega$. From the definition of torsion it follows that tor $D=d \omega$. It is straightforward to check that $[\omega, \omega]_{\mathfrak{g}_{0}}$ is $D$-parallel. Equation 7.1(1) then gives

$$
D_{Z} \text { tor } D=D_{Z} \Omega, \quad Z \in \Gamma(T P) \text {. }
$$

Specializing Corollary 5.4 to this case, we obtain:

Theorem C. Let $\pi: P \rightarrow M$ be a Cartan geometry with arbitrary model data, and $\omega$ the corresponding classical Cartan connection. Let $D$ denote the corresponding flat affine connection on $P$. Let $(\mathfrak{g}, \nabla)$ denote the corresponding Cartan algebroid described above. Then $(\mathfrak{g}, \nabla)$ is locally symmetric (in the sense of Sect.2.5) if and only if the curvature $\Omega$ of the Cartan geometry, viewed as a TP-valued two-form on $P$, is D-parallel.

\section{REFERENCES}

[1] D. V. Alekseevsky and P. W. Michor. Differential geometry of Cartan connections. Publ. Math. Debrecen, 47(3-4):349-375, 1995. MR1362298 (96h:53029)

[2] A. D. Blaom. Lie algebroids and Cartan's method of equivalence. In preparation, 2005.

[3] A. Cannas da Silva and A. Weinstein. Geometric Models for Noncommutative Algebras, volume 10 of Berkeley Mathematics Lecture Notes. American Mathematical Society, Providence, RI, 1999. MR:1747916 (2001m:58013)

[4] A. Cap and A. R. Gover. Tractor calculi for parabolic geometries. Trans. Amer. Math. Soc., 354(4):1511-1548 (electronic), 2002. MR1873017(2003j:53033)

[5] M. Crainic and R. L. Fernandes. Integrability of Lie brackets. Ann. of Math. (2), 157(2):575620, 2003. MR1973056(2004h:58027)

[6] M. Crainic and R. L. Fernandes. Secondary characteristic classes of Lie algebroids. Quantum Field Theory and Noncommutative Geometry, volume 662 of Lecture Notes in Physics. Springer, Berlin, 2005, 157-176. MR2179182

[7] P. Dazord. Groupoïde d'holonomie et géométrie globale. C. R. Acad. Sci. Paris Sér. I Math., 324(1):77-80, 1997. MR:1435591 (97m:58214)

[8] R. L. Fernandes. Lie algebroids, holonomy and characteristic classes. Adv. Math., 170(1):119179, 2002. MR1929305 (2004b:58023)

[9] R. B. Gardner. The Method of Equivalence and its Applications. SIAM, Philadelphia, 1989. MR1062197 (91j:58007)

[10] P. Griffiths. On the theory of variation of structures defined by transitive, continuous pseudogroups. Osaka J. Math., 1:175-199, 1964. MR0180993(31:5223)

[11] P. J. Higgins and K. C. H. Mackenzie. Algebraic constructions in the category of Lie algebroids. J. Algebra, 129:194-230, 1990. MR1037400 (92e:58241) 
[12] S. Kobayashi. Transformation Groups in Differential Geometry. Springer-Verlag, New York, 1972. MR0355886 (50:8360)

[13] A. Kumpera and D. Spencer. Lie Equations. Vol. I: General Theory. Princeton University Press, Princeton, N.J., 1972. MR0380908 (52:1805)

[14] K. C. H. Mackenzie. General Theory of Lie Groupoids and Lie Algebroids, volume 213 of London Mathematical Society Lecture Note Series. Cambridge University Press, Cambridge, 2003. MR2157566

[15] J. E. Marsden and T. S. Ratiu. Introduction to Mechanics and Symmetry, volume 17 of Texts in Applied Mathematics. Springer, 1994. MR1304682 (95i:58073)

[16] R. W. Sharpe. Differential Geometry: Cartan's generalization of Klein's Erlangen program, volume 166 of Graduate Texts in Mathematics. Springer, 1997. MR1453120 (98m:53033)

[17] J. Slovák. Parabolic Geometries. Research Lecture Notes, Masaryk University, 1997.

Department of Mathematics, University of Auckland, Private Bag 92019, Auckland, New ZeALAND 